\title{
$\beta$-catenin mediates the effect of GLP-1 receptor agonist on ameliorating hepatic steatosis induced by high fructose diet
}

\author{
Zhe Gao, ${ }^{1,2}$ Guang-Yao Song, ${ }^{1,2}$ Lu-Ping Ren, ${ }^{2}$ Hui-Juan Ma, ${ }^{2}$ Bo-Qing Ma, ${ }^{2}$ Shu-Chun Chen ${ }^{2}$ \\ ${ }^{1}$ Department of Internal Medicine, Hebei Medical University, Shijiazhuang, Hebei \\ ${ }^{2}$ Department of Endocrinology, Hebei General Hospital, Shijiazhuang, Hebei, China
}

\begin{abstract}
The hypoglycemic drug GLP-1 receptor agonist can ameliorate hepatic steatosis but the mechanism is not clear. Intake of high fructose leads to non-alcoholic fatty liver disease by stimulating lipid synthesis, and $\beta$-catenin is the key molecule for realizing GLP-1 function in extrahepatic tissues; with the discovery of GLP-1 receptor in liver, we speculate that $\beta$-catenin might mediate GLP-1 receptor agonist on ameliorating hepatic steatosis induced by high fructose. Wistar rats were fed with high fructose diet for 8 weeks and then treated with GLP-1 receptor agonist exenatide for 4 weeks; the changes of lipid synthesis pathway factors, the expression and nuclear translocation of $\beta$-catenin, and the hepatic steatosis of the rats were observed. After the intervention of exenatide, the hepatic steatosis induced by high fructose was improved, the nuclear translocation and expression of $\beta$-catenin were facilitated, and the mRNA and protein expression of the upstream regulator SREBP-1 and the downstream key enzymes ACC, FAS and SCD-1 of de novo lipogenesis were down-regulated. GLP-1 receptor agonist may ameliorate hepatic steatosis induced by high fructose by $\beta$-catenin regulating de novo lipogenesis pathway. GLP-1 receptor agonist may be a potential new drug for the treatment of non-alcoholic fatty liver disease, and the $\beta$-catenin may be an important target for the drug therapy.
\end{abstract}

Key words: Fructose; hepatic steatosis; GLP-1 receptor agonist; $\beta$-catenin; de novo lipogenesis.

Correspondence: Guang-Yao Song, Department of Internal Medicine, Hebei Medical University, Shijiazhuang, Hebei, 050017, China; Hebei General Hospital, Shijiazhuang, Hebei, 050051, China.

E-mail: sguangyao@163.com

Contributions: ZG, GYS, carried out the studies, participated in collecting the data, drafted the manuscript and were responsible and accountable for the accuracy and integrity of the work; LPR, HJM, BQM, SCC, performed the statistical analysis and participated in the study design. All the authors have read and approved the final version of the manuscript and agreed to be accountable for all aspects of the work.

Conflict of interest: The authors declare that they have no competing interests, and all authors confirm accuracy.

Availability of data and materials: All data generated or analyzed during this study are included in this published article.

Ethical Approval: The study was approved by the Animal Ethics Committee of Hebei General Hospital.

Patient consent for publication: Not applicable

Informed consent: Not applicable. 


\section{Introduction}

Non-alcohol fatty liver disease (NAFLD) is a metabolic stress liver injury characterized by hepatic steatosis and closely related to insulin resistance and genetic susceptibility. Epidemiological data have confirmed that non-alcoholic fatty liver disease has become one of the most important public health problems in the world in the $21^{\text {st }}$ century, ${ }^{1}$ and there is still no special effect treatment, and new drugs targeting the mechanisms of its occurrence and development are yet to be developed.

The occurrence of NAFLD is related to bad dietary habits, especially the high intake of fructose. ${ }^{2}$ In recent years, the consumption of cola and juice has been increasing significantly. It is of practical significance to establish an animal model of hepatic steatosis by using high fructose diet, to observe the effect of drugs thereon. In the previous study of our research group, high fructose can induce hepatic steatosis, and it is found that increased endogenous triglyceride (TG) production in the liver is the main mechanism of high fructose-induced liver lipid deposition.,

Gastric inhibitory polypeptide (GIP) and glucagon like peptide-1 (GLP-1) are two main active peptides for incretin. The development of GLP-1 receptor agonist as a new hypoglycemic drug attracts increasing attention. It has been found that GLP-1 receptor is widely distributed in human body. In 2010, it was first confirmed the existence of GLP-1 receptor in human primary hepatocyte,${ }^{5}$ which provided a biological basis for the study of the interaction between GLP-1 and liver. Since then, clinical and basic studies have found that GLP-1 receptor agonist can ameliorate hepatic steatosis, but the specific mechanism is not clear.,

The function of $\beta$-catenin is mainly to mediate cell adhesion and participate in gene expression. In recent years, it has been found that $\beta$-catenin is a multifunctional protein, which is also closely related to obesity, diabetes, non-alcoholic fatty liver disease and metabolic syndrome. ${ }^{8}$ With the deepening of the study, it is found that $\beta$-catenin plays an important role in the function of GLP-1. ${ }^{9}$ GLP-1 regulates pancreatic $\beta$ cell to improve glucose metabolism through $\beta$-catenin pathway which may have the function of $\beta$-catenin phosphorylation and nuclear import by activating cAMP/PKA after binding GLP-1 to GLP-1 receptor. ${ }^{10} \beta$-catenin also mediates the effect of GLP-1 on lipogenesis. Animal experiments showed that the content of TG in hepatocyte of mice after knocking out $\beta$-catenin gene increased. ${ }^{11}$ In recent years, it has been found that there is a GLP-1 receptor in the liver, which is a G protein-coupled receptor. Whether GLP-1 can activate $\beta$-catenin nuclear import in the same way of binding receptor to play a role in improving liver lipid deposition has not been studied. Therefore, this study used high fructose for inducing hepatic steatosis in Wistar rats and the rats were treated with GLP-1 receptor agonist, in order to observe the changes of de novo lipogenesis pathway and $\beta$-catenin level, to reveal the role of GLP-1 and $\beta$-catenin in the occurrence and development of non-alcoholic fatty liver disease, and to provide a theoretical basis for the search for new drug therapy for non-alcoholic fatty liver disease.

\section{Materials and Methods}

\section{Animals and grouping}

The investigation was performed on 72 cleaning 6-week-old male Wistar rats with body weight of about $200 \mathrm{~g}$, purchased from the Experimental Animal Center of Hebei Medical University (Shijiazhuang, China); drinking water was available all the time, room temperature was around $25^{\circ} \mathrm{C}$, relative humidity was in the range of $40 \sim 70 \%$, illumination was maintained for $12 \mathrm{~h}$ every day, diurnal cycle. After one week of adaptive feeding, the rats were randomly divided into normal diet group (ND) and high fructose diet group (HFD). The ND group was fed with common feed (Experimental Animal Center of Hebei Medical University, Shijiazhuang, China), and the HFD group was fed with high fructose diet - food grade crystalline fructose (Hebei Huaxu Pharmaceutical Co., Ltd., Shijiazhuang, China) was added on the basis of normal diet, such that the fructose accounted for $60 \%$ of the total calories. Eight weeks later, six rats were randomly selected from the ND group and the HFD group and killed, the liver tissues were taken to prepare light microscope specimens to confirm the establishment of the animal model of hepatic steatosis. Then the HFD group was randomly divided into HFD and exenatide group (high fructose diet group with exenatide intervention, HFD+Ex). The HFD+Ex group received exenatide $(10 \mu \mathrm{g} / \mathrm{kg}$, Eli Lilly and Co., Indianapolis, IN, USA) subcutaneous injection twice a day; the ND group and the HFD group were given the same volume of saline. After four weeks of drug intervention, all the rats fasted for $8 \mathrm{~h}$ and were killed after anesthesia with $3 \%$ pentobarbital sodium to collect blood and tissue.

\section{Intraperitoneal glucose tolerance test}

Fasting blood glucose ( 0 min blood glucose) was measured in tail blood of rats after overnight fasting for $12 \mathrm{~h}$, and then $50 \%$ glucose injection $(2 \mathrm{~g} / \mathrm{kg})$ was injected intraperitoneally; blood glucose was measured at 5, 10, 30, 60 and 120 min after giving glucose, and the glucose area under the curve $\left(\mathrm{AUC}_{\mathrm{glu}}\right)$ of each rat was calculated.

\section{Hyperinsulinemic-euglycemic clamp test in waking state}

The rats were anesthetized with $3 \%$ pentobarbital sodium and the right jugular vein and right carotid artery were intubated. After the body weight of the rats returned to the preoperative level and the stress reaction disappeared, the hyperinsulinemic-euglycemic clamp test was performed in waking state. One end of the T-pipe intubated in the jugular vein was connected with $40 \mathrm{mU} / \mathrm{mL}$ insulin injection, and the other end was connected with $30 \%$ glucose injection. The basic blood glucose was measured first, and then insulin solution was infused at a fixed rate of $4 \mathrm{mU}(\mathrm{kg} / \mathrm{min})$. When the blood glucose decreased below the base value, the glucose injection was infused synchronously at the rate of $9 \sim 14 \mathrm{mg}$ ( $\mathrm{kg} / \mathrm{min}$ ). The glucose infusion rate (GIR) was adjusted according to the blood glucose which was stabilized within the range of $5 \pm 0.5 \mathrm{mmol} / \mathrm{L}$. GIR= waking-state glucose infusion rate $\times$ glucose concentration $\times 1000 /$ body weight $(\mathrm{kg}) / 60$; it is used to evaluate insulin sensitivity in rats.

\section{Assessment of lipid deposition in liver tissue}

Liver tissues were subjected to determination of the steatosis score and an assay of the synthesis of TG. The change in steatosis score from baseline was assessed (steatosis grade $0=<5 \%$ steatosis, $1=5-33 \%$ steatosis, $2=34-66 \%$ steatosis, $3=>66 \%$ steatosis $)$. The content of TG in liver was determined by GPO-PAP method according to the operation process provided by triglyceride determination kit (Pulilai Bioengineering Institute, Beijing, China).

\section{Oil-red-O staining in liver tissue}

Frozen sections of liver tissue with a thickness of about $8 \mu \mathrm{m}$ were fixed with $10 \%$ neutral formalin for $10 \sim 15 \mathrm{~min}$, and then washed. After applying Oil-red-O (Sigma-Aldrich, St. Louis, MO, USA) staining for $10 \sim 15 \mathrm{~min}$, they were decolorized with $60 \%$ isopropyl alcohol. After that, hematoxylin was used for slightly staining the nucleus, then it was photographed. 


\section{Immunohistochemistry}

Fresh liver tissue was fixed by $10 \%$ neutral formalin, dehydrated, embedded in paraffin, and sliced into slices with a thickness of about $3 \mu \mathrm{m}$ by a microtome. The slides were dewaxed, then internal peroxidase was inactivated with $3 \%$ hydrogen peroxide in $100 \%$ methanol for $30 \mathrm{~min}$. Antigen retrieval was performed with microwave oven in $10 \mathrm{mM}$ citrate buffer for $15 \mathrm{~min}$. Ten percent normal goat serum in PBS was added to the sections for $30 \mathrm{~min}$ at room temperature to block nonspecific antibody binding. After that, the sections were incubated with primary antibody for $\beta$ catenin (diluted 1:200, Cell Signaling Technology) overnight at $4^{\circ} \mathrm{C}$. After rinsing in PBS, biotinylated secondary antibody (diluted 1:1000, Kangwei Century Biotechnology Co., Ltd., Beijing, China) was added and incubated for $30 \mathrm{~min}$ at room temperature. Horseradish peroxidase labeled streptomyces albumin working solution (S-A/HRP) was added, using DAB for color development and hematoxylin for counterstaining.

\section{Extraction of nuclear protein from liver}

The liver tissue samples were accurately weighed and cut into small pieces and operated according to the nuclear protein extraction kit (Pulilai Gene Technology Co., Ltd., Beijing, China). Cytosol Extraction Buffer A (CEB-A) was added, and a glass homogenizer was used to break the tissue on ice. The split product was oscillated repeatedly and violently, after ice bath, then the Cytosol Extraction Buffer B (CEB-B) was added. After being centrifuged at $4^{\circ} \mathrm{C}$, the precipitate was the nuclear crude extract. CEB$\mathrm{A}$ and CEB-B were added in proportion for repeated oscillation and ice bath, centrifuging at $4^{\circ} \mathrm{C}$ to discard the supernatant (this step was repeated), then the pre-cooled Nuclear Extraction Buffer (NEB) was added to the centrifugal precipitate. After drastic oscillation and ice bath, and centrifugation at $4{ }^{\circ} \mathrm{C}$, the supernatant was taken for preservation.

\section{Gene expression of liver lipid metabolism related factors}

The total RNA was extracted by Trizol and the mRNA expression of $\beta$-catenin and lipid metabolism related genes including sterol regulatory element binding protein-1 (Srebp-1), acetyl-CoA carboxylase $(A c c)$, fatty acid synthase $(\mathrm{Fas})$ and stearoyl-CoA desaturase-1 $(S c d-1)$ in liver tissues was detected by real-time fluorescence quantitative PCR (RT-PCR). The primers were designed using DNAMAN software, and were synthesized by Shanghai BioEngineering Co., Ltd. using the American PE company's 391 DNA automatic synthesizer. The sequence of primers was shown in Table 1.

\section{Determination of protein expression in liver}

Western blot was used to determine the protein expression levels of SREBP-1 (Santa Cruz Biotechnology Inc., Dallas, TX, USA), and downstream-lipid-synthesis key enzymes FAS (Cell Signaling Technology Inc., Danvers, MA, USA), ACC (Cell Signaling Technology), SCD-1 (Abcam, Cambridge, MA, USA) and $\beta$-catenin (Cell Signaling Technology); the references were $\beta$ actin (Cambridge Bioscience, Cambridge, UK) and LIMN1 (BBI Life Sciences, Shanghai, China). Total protein and nuclear protein of rat liver tissue were extracted. $50 \mu \mathrm{g}$ protein was taken for SDSPAGE gel electrophoresis, transferred membrane, blocked, then the antibody diluted at 1:1000 1:2000 was added and shaken in a table concentrator at $4^{\circ} \mathrm{C}$ overnight. After being washed with TTBS buffer and added a 1:10000 diluted horseradish peroxidaseconjugated secondary antibody to the membrane for $1 \mathrm{~h}$, it was subjected to chemiluminescence, development, fixation and semiquantitative analysis of protein expression.

\section{Statistical analysis}

All the data were processed by SPSS13.0 and the data results were expressed by as mean $\pm \mathrm{SD}$. The one-way analysis of variance of complete random design was used for the comparison of multiple groups, the Least Significant Difference test was used for the pairwise comparison. Abnormally distributed data among groups was analyzed with the Kruskal-Wallis one-way analysis of variance method. $\mathrm{P}<0.05$ meant the difference was statistically significant.

\section{Results}

\section{GLP-1 receptor agonist improved body weight, liver index and maintained glucose homeostasis in rats}

After high-fructose feeding for 8 weeks, the body weight and hepatic index [hepatic index $(\%)=$ (hepatic wet weight $/$ body weight $\times 100 \%$ ] of HFD group were significantly higher than those of ND group $(\mathrm{P}<0.05)$; after intervention with Exenatide for 4 weeks, the body weight and hepatic index in HFD+Ex group were notably lower than those in HFD group $(\mathrm{P}<0.05$, Figure 1 A,B). The results of IPGTT compared with ND group showed that the $0,5,30,60,120 \mathrm{~min}$ blood glucose and $\mathrm{AUC}_{\mathrm{glu}}$ increased in HFD group $(\mathrm{P}<0.05)$. After intervention with exenatide, the 0,30 , 60, 120 min blood glucose and $\mathrm{AUC}_{\mathrm{glu}}$ significantly decreased in HFD+Ex group compared with those in the HFD group $(\mathrm{P}<0.05$, Figure 1 C,D), which showed glucose tolerance was improved. GIR in HFD group was prominently lower than that in ND group $(\mathrm{P}<0.05)$, while GIR in HFD+Ex group was higher than that in HFD group $(\mathrm{P}<0.05$, Figure $1 \mathrm{E})$, which reflected that GLP-1 receptor agonist improved insulin resistance induced by high fructose diet.

\section{GLP-1 receptor agonist improved lipid profile and liver enzymes in rats}

Compared with ND group, TG and FFA increased in HFD group $(\mathrm{P}<0.05$, Figure $2 \mathrm{~A}, \mathrm{C})$, no significant difference was found in TC $(\mathrm{P}>0.05$, Figure $2 \mathrm{~B})$. Compared with HFD group, TG and FFA decreased in HFD+Ex group $(\mathrm{P}<0.05)$, suggesting that GLP1 receptor agonist improved lipid profile in rats. At the same time, ALT increased in HFD group $(\mathrm{P}<0.05)$, ALT decreased after treatment with Exenatide ( $\mathrm{P}<0.05$, Figure $2 \mathrm{D})$, but there was no significant difference in AST among three groups ( $\mathrm{P}>0.05$, Figure $2 \mathrm{E})$.

\section{GLP-1 receptor agonist improved the hepatic steatosis induced by high fructose}

Compared with ND group, TG concentration and steatosis score of the rat liver in HFD group increased $(\mathrm{P}<0.05)$, and a large number of red lipid droplets were found in hepatocytes (Figure 3 A-C), suggesting that fructose induced hepatic steatosis. After treatment with exenatide, the concentration of TG and steatosis

Table 1. Primers sequences of rats used for Real Time Quantitative PCR.

\begin{tabular}{lll} 
Primer & Forward & Reverse \\
Gapdh & TGAACGGGAAGCTCACTGG & GCTTCACCACCTTCTTGATGTC \\
Srebp-1 & GCTTCTCTGGGCTCCTCTCT & GCACTGGCTCCTCTTTGATT \\
\hline Acc & TCCCGCACCTTCTTCTACTG & ACTTCCACAAACCAGCGTCT \\
Fas & TGAAGACATCCTTGAGCCTTG & ATTCATTGGCACACTTTCAGG \\
\hline Scd-1 & CAGTTCCTACACGACCACCA & GGACGGATGTCTTCTTCCAG \\
$\beta$ - catenin & GCTTGTTGGCCATCTTTAAATC & ACAGTTTTGAACAAGTCGCTGA \\
\hline
\end{tabular}


score in HFD+Ex group were lower than that in HFD group $(\mathrm{P}<0.05$, Figure $3 \mathrm{~A}, \mathrm{~B})$, and the red lipid droplets decreased (Figure $3 \mathrm{C}$ ), suggesting that GLP-1 receptor agonist improved hepatic steatosis induced by high fructose.

GLP-1 receptor agonist inhibited the expression of factors related to lipid synthesis pathway activated by high fructose

Compared with ND group, the mRNA and protein expression levels of upstream transcription factor SREBP-1 (Figure 4 A,C) and key enzymes of downstream lipid synthesis ACC, FAS and SCD-1 (Figure 4 B,D) significantly increased in rat liver tissue of HFD group, suggesting that fructose activated the de novo lipogenesis pathway. Compared with HFD group, the expressions of SREBP-1, ACC, FAS and SCD-1 were down-regulated in HFD+Ex group after intervention with exenatide, suggesting that GLP-1 receptor agonist improved hepatic steatosis induced by high fructose by inhibiting the de novo lipogenesis.

\section{GLP-1 receptor agonist promoted the expression and nuclear translocation of $\boldsymbol{\beta}$-catenin}

Compared with ND group, the expression of $\beta$-catenin in rat liver tissue of HFD group decreased $(\mathrm{P}<0.05)$; compared with HFD group, the expression of mRNA, total protein and nucleoprotein of $\beta$-catenin in HFD+Ex group increased after intervention with Exenatide $(\mathrm{P}<0.05$, Figure $5 \mathrm{~A}-\mathrm{C})$. Immunohistochemistry showed that the HFD+Ex group had more nuclear $\beta$-catenin staining than the HFD group (Figure 5D) after exenatide intervention, suggesting $\beta$-catenin nuclear translocation in hepatocytes.

\section{Discussion}

In recent years, with the changes of dietary structure and lifestyle of people, the incidence of non-alcoholic fatty liver disease is increasing year by year. ${ }^{1}$ Although many basic and clinical studies have been carried out on NAFLD, the pathogenesis of NAFLD has not yet been fully clarified, and there is also a lack of effective drugs in the treatment. Therefore, it is of importance to establish an animal model similar to the incidence of human NAFLD, to explore its pathogenesis, and to find and select effective therapeutic drugs. The results of this study show that high fructose diet can induce hepatic steatosis in rats, and GLP-1 receptor agonist may ameliorate hepatic steatosis induced by high fructose by $\beta$-catenin regulating de novo lipogenesis pathway.

The novel hypoglycemic drug GLP-1 receptor agonist can promote insulin synthesis in a glucose dependent manner, and it can also bind to receptors on $\alpha$ cells to inhibit glucagon secretion, so as to regulate blood glucose levels. In this study, we selected the GLP-1 receptor agonist exenatide as the experimental intervention drug, and the glucose homeostasis was maintained after medications for four weeks which was consistent with the results of previous studies. ${ }^{12}$ During the treatment of diabetes mellitus, it has been found that GLP-1 receptor agonists such as exenatide and liraglutide have shown good effects in reducing glucose, losing weight and improving insulin resistance, and the effect of treatment has aroused people's attention for patients with diabetes and NAFLD. ${ }^{6}$ A number of clinical trials have shown that GLP-1 receptor agonist improved liver enzymology and histology in patients with NAFLD. ${ }^{13,14}$
A

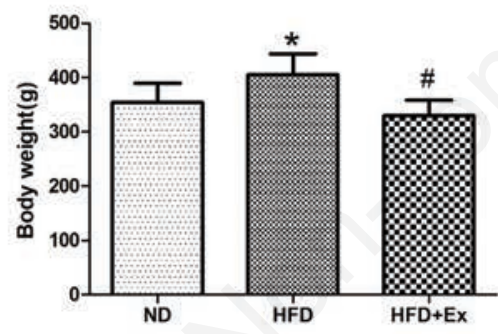

B

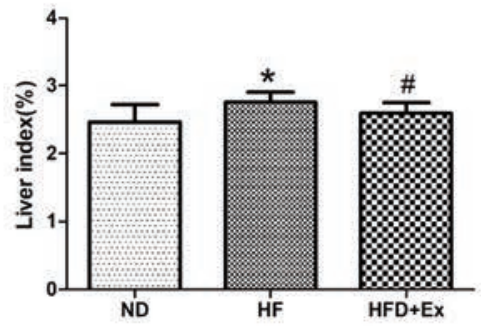

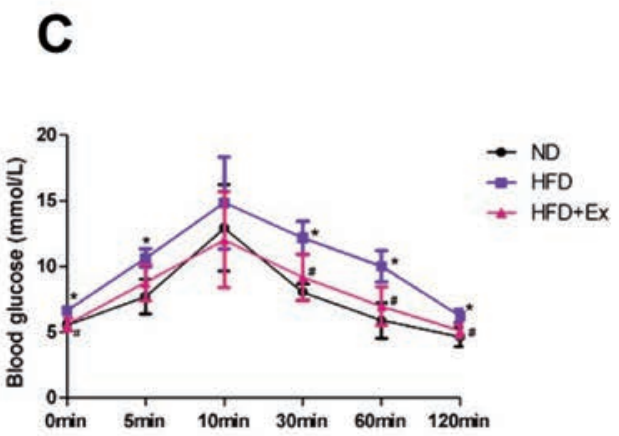
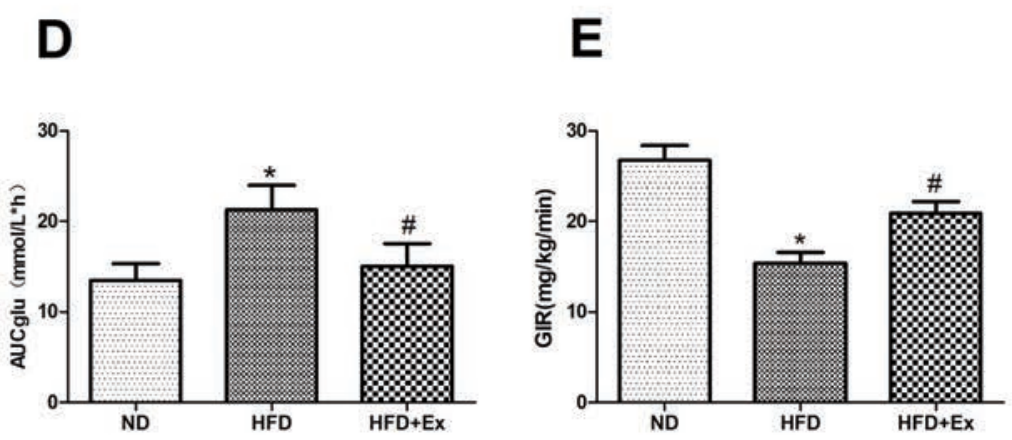

Figure 1. GLP-1 receptor agonist alleviated body weight, liver index and glucose homeostasis in rats. A) Body weight; B) liver index; C) intraperitoneal glucose tolerance test (IPGTT); D) area under curve of glucose; E) euglycemic hyperinsulinemic clamp test in three groups ( $\mathrm{n}=6$ per group) after 4-week drug administration. Results are expressed as the mean $\pm \mathrm{SD}{ }^{*} \mathrm{P}<0.05$ vs $\mathrm{ND}$; \# $<<0.05$ vs $\mathrm{HFD}$. $\mathrm{ND}$, normal diet; HFD, high fructose diet; HFD+Ex, high fructose diet group with exenatid intervention; $\mathrm{AUC}_{\mathrm{glu}}$, area under the curve; GIR, glucose infusion rate. 
It has also been reported that exenatide could ameliorate hepatic steatosis in obese mice and $\mathrm{db} / \mathrm{db}$ mice induced by high fat diet in animal experiments. ${ }^{15,16}$ GLP-1 receptor agonists could also improve body weight, liver lipid content, serum FFA and liver enzyme levels in mice fed with high fat. ${ }^{17}$ Our study found that exenatide could ameliorate hepatic steatosis induced by high fruc- tose and reduce liver enzyme. The results showed that after intervention of four weeks with exenatide, the body weight and hepatic index of exenatide group were significantly improved compared with those of high fructose group, and the body weight was even lower than that of normal diet group.
A

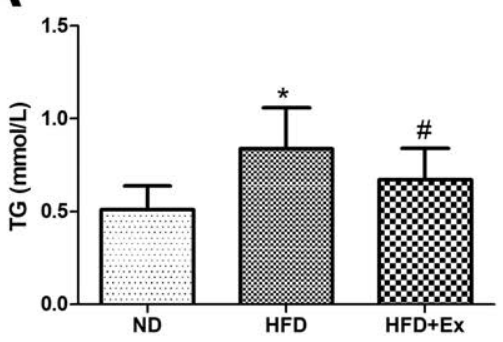

D
B

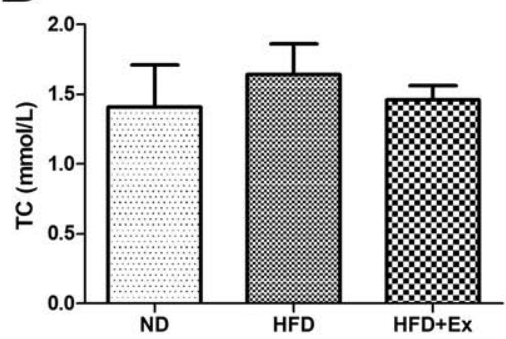

C

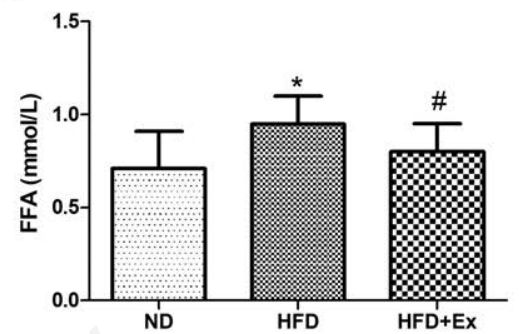

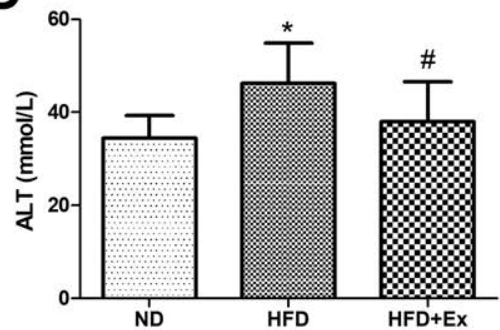

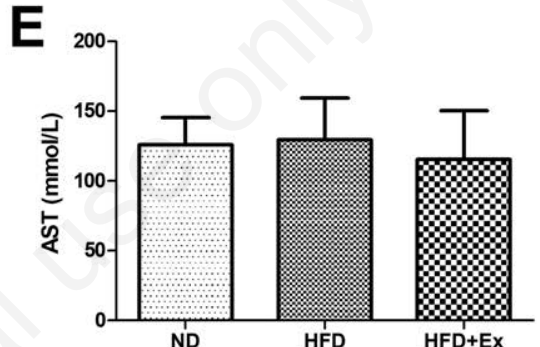

Figure 2. GLP-1 receptor agonist ameliorated lipid profile and liver enzyme in rats. Lipid profile (A-C) liver enzymes (D,E) in three groups (n=6 per group) after 4-week drug administration. Results are expressed as the mean \pm SD. ${ }^{*} \mathbf{P}<\mathbf{0 . 0 5} v$ s $\mathrm{ND}$, \#P<0.05 vs $\mathrm{HFD}$; TG, triglyceride; TC, total cholesterol; FFA, free fat acid; ALT, alanine aminotransferase; AST, aspartate.
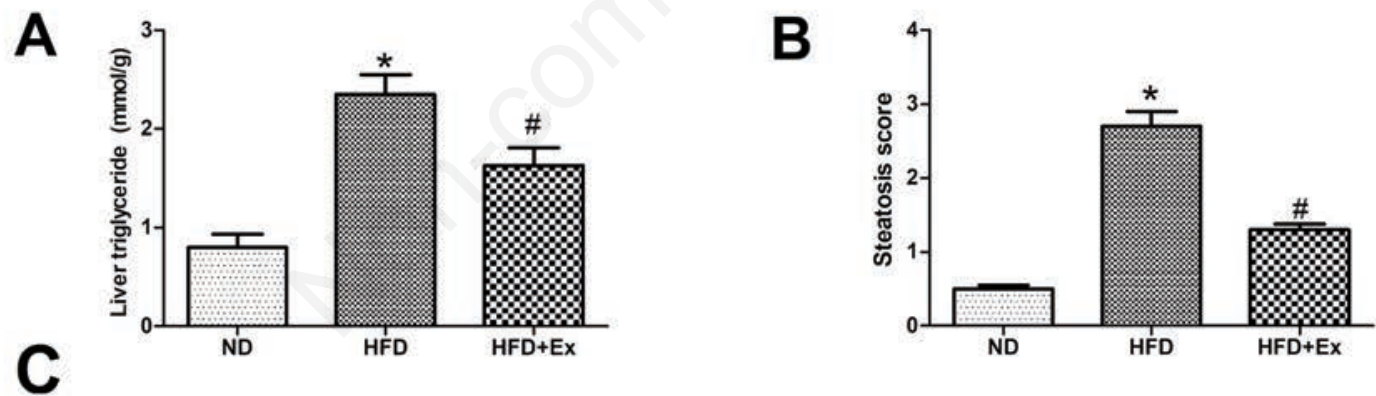

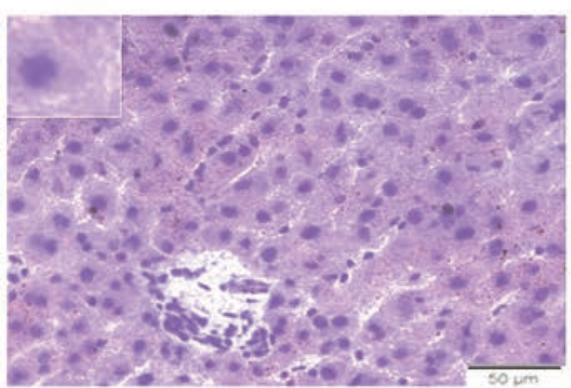

ND

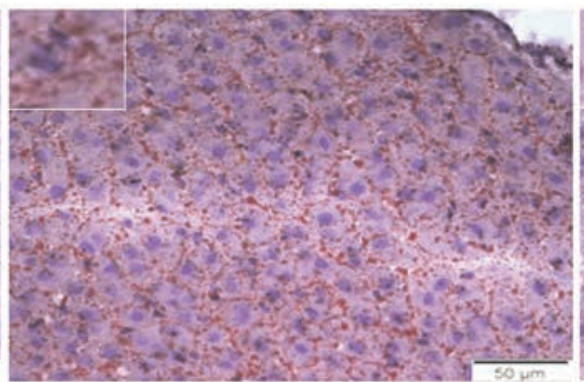

HFD

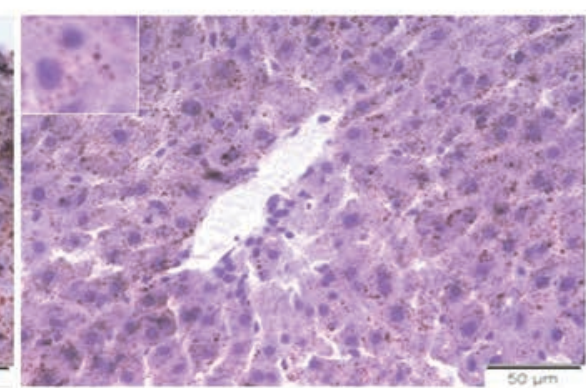

HFD+Ex

Figure 3. GLP-1 receptor agonist improved liver fat deposition induced by high fructose diet in rats. A) Triglyceride concentration of the liver was determined in the different groups. B) The change in steatosis score from baseline was assessed. C) Oil-red-O staining of liver tissues in three groups ( $n=6$ per group) after 4-week drug administration; original magnification: $\times 400 ;$ scale bar: $50 \mu$ m. Results are expressed as the mean $\pm \mathrm{SD} . * \mathrm{P}<0.05$ vs $\mathrm{ND}$, \# $\mathrm{P}<0.05$ vs $\mathrm{HFD}$. 
A

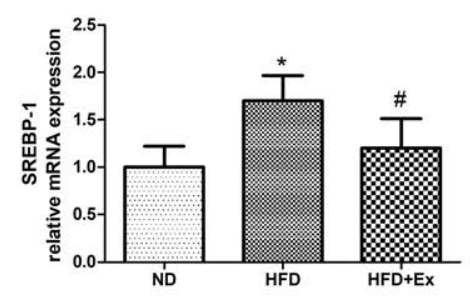

C
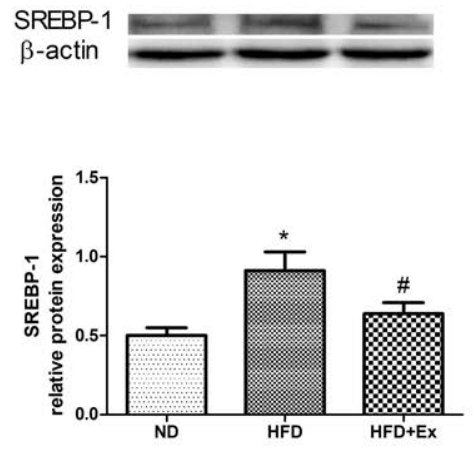

B
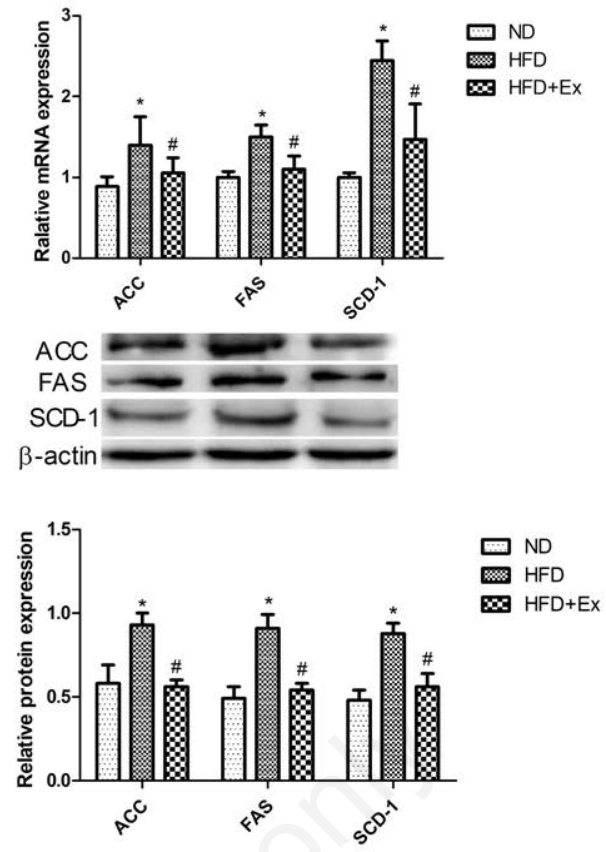

Figure 4. GLP-1 receptor agonist alleviated high-fructose-induced de novo lipogenesis in rats. A) The relative mRNA expression level of SREBP-1 in three groups ( $n=6$ per group) after 4-week drug administration. B) The relative mRNA expression levels of ACC, FAS and SCD-1 in the lipogenic pathway. C) SREBP-1 protein expression was determined by western blotting ( $n=6$ per group); $\beta$-actin served as the loading control. D) Protein expression levels of ACC, FAS and SCD-1. Results are expressed as the mean \pm SD. ${ }^{*} \mathbf{P}<0.05$ vs $\mathrm{ND}, \# \mathrm{P}<0.05$ vs $\mathrm{HFD}$; SREBP-1, sterol regulatory element-binding protein 1; ACC, acetyl-CoA carboxylase; FAS, fatty acid synthase; SCD-1, stearoyl-CoA desaturase 1 .

A

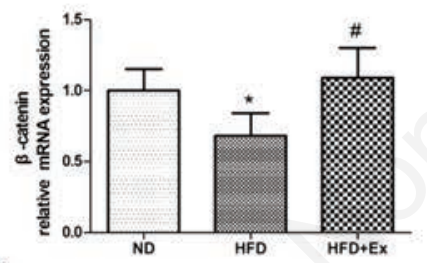

D

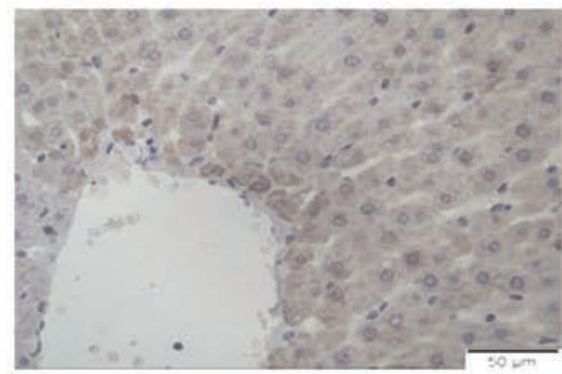

ND

B
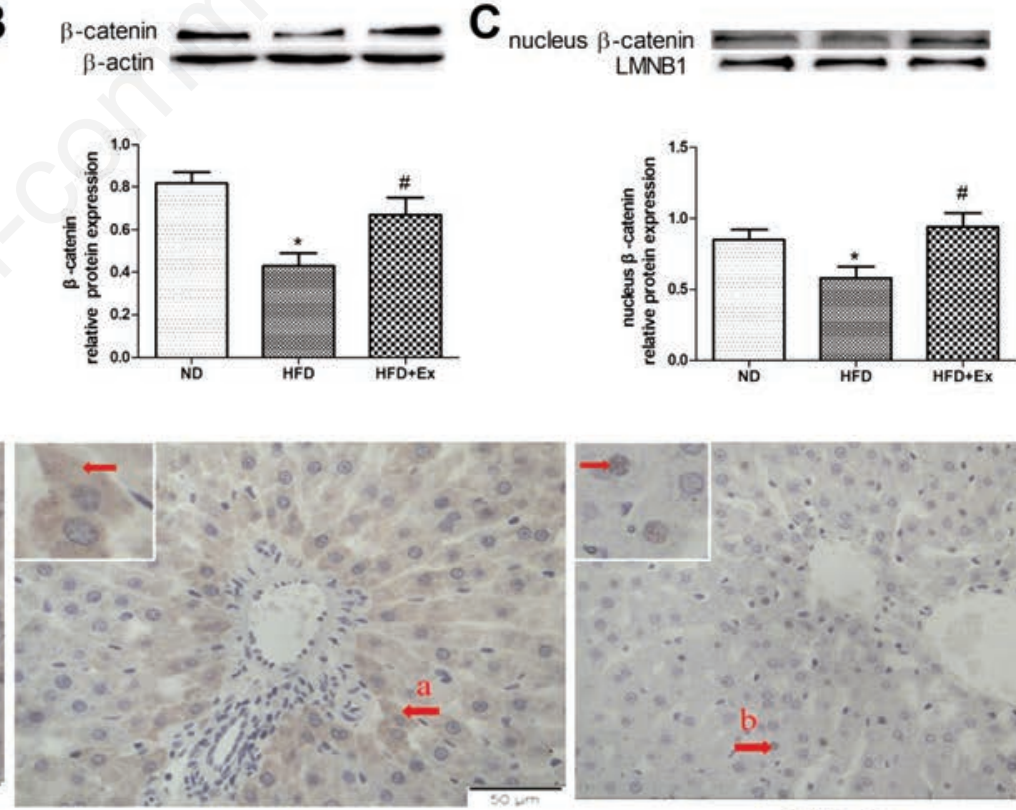

HFD

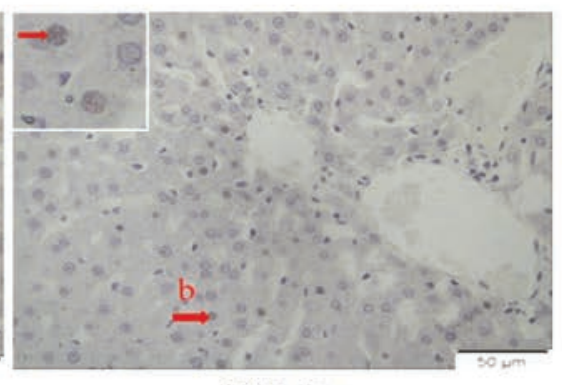

HFD+Ex

Figure 5. GLP-1 receptor agonist increased expression and nuclear transfer of $\beta$-catenin in exenatide-suppressed hepatic steatosis in rats. A) The relative $\mathrm{mRNA}$ expression level of $\beta$-catenin in three groups ( $\mathrm{n}=\mathbf{6}$ per group) after 4-week drug administration. B) $\beta$-catenin protein expression ( $n=6$ per group); $\beta$-actin served as the loading control. C) Nuclear protein expression of $\beta$-catenin, LMNB1 served as the loading control. D) Immunohistochemical staining for $\beta$-catenin in liver tissues in three groups after 4-week drug administration; original magnification: $\times 400$, scale bar: $50 \mu \mathrm{m}$;,$\beta$-catenin was shown in the cytoplasm, $b, \beta$-catenin was displayed in the nucleus. Results are expressed as the mean \pm SD. ${ }^{*} \mathrm{P}<0.05$ vs $\mathrm{ND}, \# \mathrm{P}<0.05$ vs HFD. LMNB1, lamin B1. 
The decrease of body weight and hepatic index may also be an important cause of liver histology changes, ${ }^{18}$ and it has been reported that weight loss in patients treated with GLP-1 receptor agonist was related to decrease of adipose tissues. ${ }^{19}$ In addition, we found that the decrease of serum TG and FFA levels and the improvement of glucose infusion rate suggested the improvement of lipid metabolism and insulin resistance. Insulin resistance and lipid metabolism abnormalities are the basis of NAFLD formation. Under the condition of insulin resistance, the inhibitory effect of insulin on insulin-sensitive lipase activity decreases, and the free fatty acids in vivo increase; free fatty acids are an important source of liver TG, which provides a substrate for the synthesis of TG in the liver and leads to the hepatic steatosis. In our experiment, rats were fed with the high fructose diet; when large amounts of fructose were consumed, the excessive fructose could be metabolized and converted into lipids to be stored in the liver through de novo lipogenesis for energy balance. Epidemiological studies have shown that excessive fructose intake was associated with the incidence of NAFLD. ${ }^{2}$ The core pathological basis of NAFLD is abnormal hepatic steatosis in hepatocytes, and the main mechanism of hepatic steatosis caused by high fructose intake is the increase of endogenous lipid synthesis after excessive fructose intake. ${ }^{3,20}$ In our study, high fructose successfully induced hepatic steatosis in rats, and it was found that the expressions of upstream transcription factor sterol regulatory element binding protein-1 (SREBP-1) and downstream enzymes of lipid synthesis acetyl-CoA carboxylase (ACC), fatty acid synthase (FAS) and stearoyl-CoA desaturase-1 (SCD-1) increased in rat liver in high fructose environment.

With the discovery of GLP-1 receptors in the liver, 5 it was found that the therapeutic effect of GLP-1 receptor agonists on NAFLD is not only through weight loss and improvement of insulin resistance. It was reported that administration of GLP-1 analog AC3174 did not improve liver lipid metabolism in GLP-1 receptor knockout mice, suggesting that the effect of GLP-1 on the liver depended directly on GLP-1 receptor, but its mechanism was not clear. An animal experimental study showed that the activation of GLP-1 receptor could ameliorate hepatic steatosis induced by high fat diet in mice. ${ }^{21}$ GLP-1 receptor agonists CNTO3649 and exendin-4 inhibited liver fat synthesis pathway by down-regulating the expressions of fatty acid synthase, SREBP-lc, and diacylglycerol acyltransferase. ${ }^{22}$ GLP-1 receptor agonist liraglutide could down-regulate the expression of ACC and FAS in the mice with non-alcoholic fatty liver disease. ${ }^{23}$ In our experiment, it was found that GLP-1 receptor agonists could inhibit the expressions of the key enzymes ACC, FAS and SCD-1 in the lipid synthesis pathway by down-regulating the expression of the liver transcription factor SREBP-1, which could improve the rat hepatic steatosis induced by high fructose, suggesting that the therapeutic effect of GLP-1 receptor agonist on NAFLD is not only through the benefits of reducing body weight and insulin resistance, but also directly acting on liver cells to regulate lipid synthesis. However, the effect target of GLP-1 receptor agonist is GLP-1 receptor located in cell membrane, SREBP-1 is a kind of membrane link protein located in endoplasmic reticulum. There is no research report on how GLP-1 receptor agonists regulate SREBP-1.

The $\beta$-catenin was first discovered by a German cell biologist, Walt Birchmeier, as an adhesion factor. Later, it was found that $\beta$ catenin is a multifunctional protein, which is involved in the regulation of cell adhesion, proliferation, signal transduction, metabolism and other biological processes, and which is also closely related to the occurrence and development of obesity, diabetes, nonalcoholic fatty liver disease and metabolic syndrome. The study found that liver-specific knockout of $\beta$-catenin gene mice showed liver lipid deposition. ${ }^{11}$ It was reported that the expression of $\beta$ catenin protein decreased in obese rats, and further down-regulation of $\beta$-catenin gene expression in rat hepatocytes could aggravate the hepatic steatosis induced by fatty acids. ${ }^{24}$ These studies

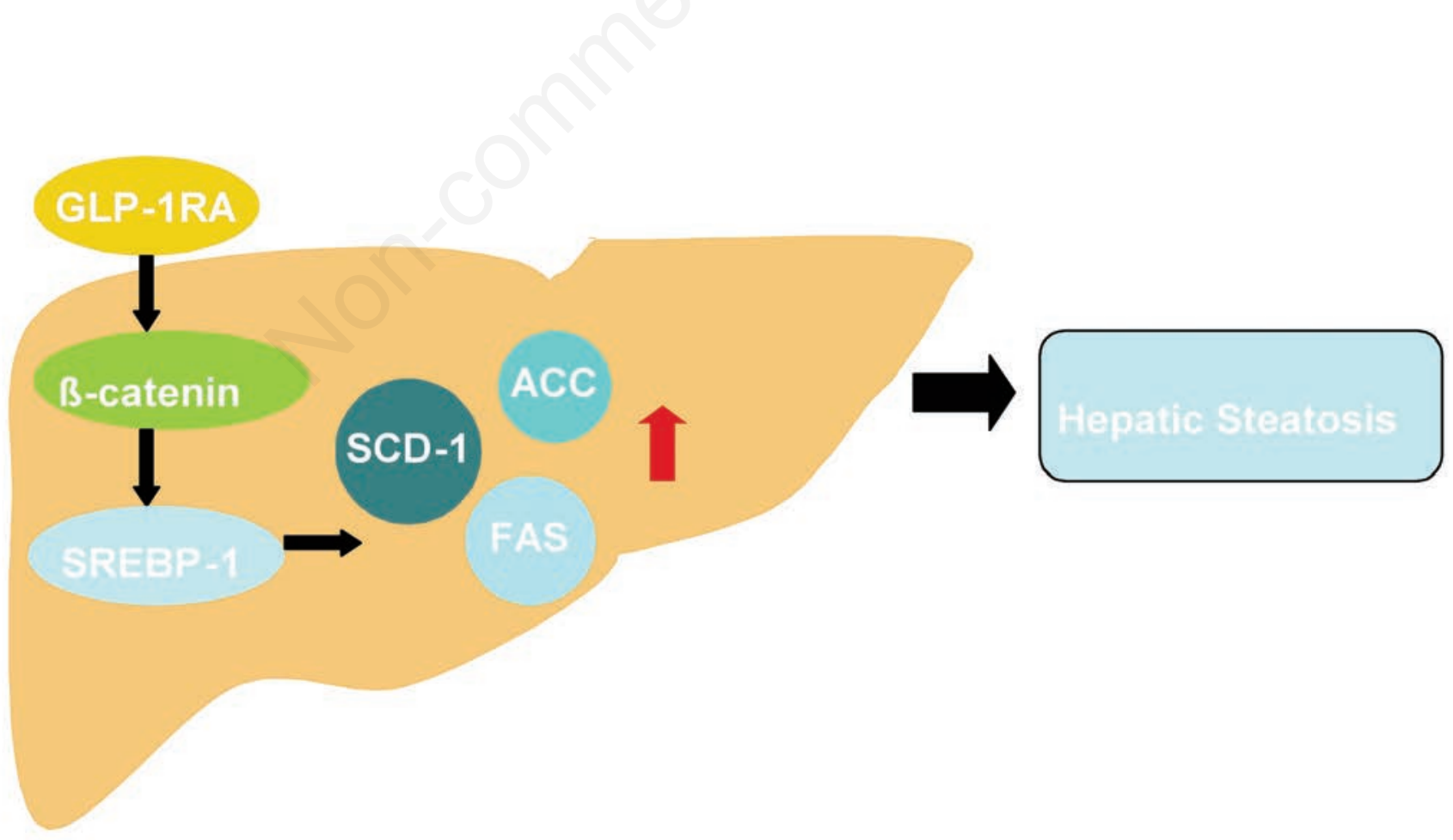

Figure 6. The schematic diagram about $\beta$-catenin mediating the effect of GLP-1 receptor agonist on ameliorating hepatic steatosis induced by high fructose diet. GLP-1RA, GLP-1 receptor agonist; SREBP-1, sterol regulatory element-binding protein 1; ACC, acetylCoA carboxylase; FAS, fatty acid synthase; SCD-1, stearoyl-CoA desaturase 1. 
suggest that $\beta$-catenin plays an important role in liver lipid metabolism. Our experiment results showed that hepatic steatosis occurred and $\beta$-catenin expression decreased in the liver of rats fed with high fructose; GLP-1 receptor agonist exenatide could increase the expression of $\beta$-catenin total protein and nucleoprotein and ameliorate hepatic steatosis, suggesting that GLP-1 receptor agonist might induce the expression of $\beta$-catenin and nuclear translocation. Previous studies have found that $\beta$-catenin plays an important role in the function of GLP-1 outside the liver. ${ }^{9}$ GLP-1 could act on the pancreas and fat through the $\beta$-catenin pathway to regulate glucose metabolism and fat production. In addition, it was found that this pathway might activate cAMP/PKA through binding GLP-1 to GLP-1 receptor, and $\beta$-catenin performed a function after phosphorylation at the Ser675 site and nuclear import. ${ }^{10}$ In recent years, GLP-1 receptors have been found in liver, ${ }^{5}$ and we speculate that GLP-1 receptor agonists may ameliorate hepatic steatosis by binding to GLP-1 receptor, to induce $\beta$-catenin to function into the nucleus in the same way. The study found that the treatment of GLP-1 receptor agonist exendin-4 could improve palmitic acid-induced steatosis of HepG2 cells, and the use of small interfering RNA to suppress the expression of $\beta$-catenin increased the expression of fat transcription factors. ${ }^{25}$ It was found that GLP-1 (28-36) could improve blood glucose, body weight and gluconeogenesis related genes of mice fed with high fat through cAMP/PKA, and the expression of $\beta$-catenin increased at the same time. ${ }^{26}$ These studies suggested that $\beta$-catenin was the key molecule for GLP-1 receptor agonist to ameliorate hepatic steatosis. Our study also found that the intervention of GLP-1 receptor agonist could induce the $\beta$-catenin expression and nuclear translocation, decrease the expression of SREBP-1 and its downstream key enzymes ACC, FAS and SCD-1, and improve the hepatic steatosis. Our experiments suggested that SREBP-1 may be an important target of $\beta$-catenin, and the relationship between $\beta$-catenin and SREBP-1 can also be traced back in previous experiments. SREBP-1 was an important transcription factor regulating the gene expression of fatty acid synthase. It has been found that Wnt10b could down-regulate SREBP-1 through $\beta$-catenin and reduce lipid accumulation in muscle cells. ${ }^{27}$ The $\beta$-catenin signaling pathway could down-regulate the fat SREBP-1 expression and inhibit the lipogenesis. ${ }^{28}$ Leptin could induce $\beta$-catenin pathway to reduce the expression of SREBP-1 in liver. ${ }^{29}$ These studies suggested that $\beta$ catenin was closely related to SREBP-1.

The current study demonstrates that GLP-1 receptor agonists may regulate the expression of SREBP-1 in liver through $\beta$-catenin and regulate the expressions of the downstream key enzymes ACC, FAS and SCD-1 in de novo lipogenesis pathway, so as to ameliorate hepatic steatosis induced by high fructose (Figure 6). Our study indicates, for the first time to our knowledge, that $\beta$-catenin is essential for the GLP-1 receptor agonist to reduce hepatic steatosis in mice, which suggests that GLP-1 receptor agonist may be a potential new drug for the treatment of non-alcoholic fatty liver disease, and the $\beta$-catenin may be an important target for the drug therapy.

\section{Acknowledgements}

The authors would like to thank the staff of Department of Hebei Key Laboratory of Metabolic Disease for their support of this research.

\section{References}

1. Benedict M, Zhang X. Non-alcoholic fatty liver disease: An expanded review. World J Hepatol 2017;9:715-32.

2. Jensen T, Abdelmalek MF, Sullivan S, Nadeau KJ, Green M, Roncal C, et al. Fructose and sugar: A major mediator of nonalcoholic fatty liver disease. J Hepatol 2018;68:1063-75.

3. Yu X, Ren LP, Wang C, Zhu YJ, Xing HY, Zhao J, Song GY, et al. Role of X-box binding protein- 1 in fructose-induced de novo lipogenesis in HepG2 cells. Chin Med J (Engl) 2018;131:2310-9.

4. Shi LJ, Shi L, Song GY, Zhang HF, Hu ZJ, Wang C, et al. Oxymatrine attenuates hepatic steatosis in non-alcoholic fatty liver disease rats fed with high fructose diet through inhibition of sterol regulatory element binding transcription factor 1 (Srebf1) and activation of peroxisome proliferator activated receptor alpha (Ppar $\alpha$ ). Eur J Pharmacol 2013;714:89-95.

5. Gupta NA, Mells J, Dunham RM, Grakoui A, Handy J, Saxena $\mathrm{NK}$, et al. Glucagon-like peptide-1 receptor is present on human hepatocytes and has a direct role in decreasing hepatic steatosis in vitro by modulating elements of the insulin signaling pathway. Hepatology 2010;51:1584-92.

6. Kalogirou M, Sinakos E. Treating nonalcoholic steatohepatitis with antidiabetic drugs: Will GLP-1 agonists end the struggle? World J Hepatol 2018;10:790-4.

7. Bifari F, Manfrini R, Dei Cas M, Berra C, Siano M, Zuin M, et al. Multiple target tissue effects of GLP-1 analogues on nonalcoholic fatty liver disease (NAFLD) and non-alcoholic steatohepatitis (NASH). Pharmacol Res 2018;137:219-29.

8. Pedone E, Marucci L. Role of $\beta$-catenin activation levels and fluctuations in controlling cell fate. Genes (Basel) 2019; 10:176.

9. Chiang YT, Ip W, Jin T. The role of the Wnt signaling pathway in incretin hormone production and function. Front Physiol 2012;3:273.

10. Xiong X, Shao W, Jin T. New insight into the mechanisms underlying the function of the incretin hormone glucagon-like peptide- 1 in pancreatic $\beta$-cells: the involvement of the Wnt signaling pathway effector $\beta$-catenin. Islets 2012;4:359-65.

11. Behari J, Yeh TH, Krauland L, Otruba W, Cieply B, Hauth B, et al. Liver-specific beta-catenin knockout mice exhibit defective bile acid and cholesterol homeostasis and increased susceptibility to diet-induced steatohepatitis. Am J Pathol 2010;176:744-53.

12. Xu F, Li Z, Zheng X, Liu H, Liang H, Xu H, et al. SIRT1 mediates the effect of GLP-1 receptor agonist exenatide on ameliorating hepatic steatosis. Diabetes 2014;63:3637-46.

13. Dong Y, Lv Q, Li S, Wu Y, Li L, Li J, et al. Efficacy and safety of glucagon-like peptide-1 receptor agonists in non-alcoholic fatty liver disease: A systematic review and meta-analysis. Clin Res Hepatol Gastroenterol 2017;41:284-95.

14. Sheriba N, Samy M, Adel C, Anas S. Relationship between glucagon like peptide-1 and non-alcoholic fatty liver disease in diabetic and non-diabetic patients. Diabetes Metab Syndr 2019;13:526-9.

15. Samson SL, Sathyanarayana P, Jogi M, Gonzalez EV, Gutierrez A, Krishnamurthy R, et al. Exenatide decreases hepatic fibroblast growth factor 21 resistance in non-alcoholic fatty liver disease in a mouse model of obesity and in a randomised controlled trial. Diabetologia 2011;54:3093-100.

16. Yamamoto T, Nakade Y, Yamauchi T, Kobayashi Y, Ishii N, Ohashi T, et al. Glucagon-like peptide-1 analogue prevents nonalcoholic steatohepatitis in non-obese mice. World J Gastroenterol 2016;22:2512-23.

17. Trevaskis JL, Griffin PS, Wittmer C, Neuschwander-Tetri BA, 
Brunt EM, Dolman CS, et al. Glucagon-like peptide-1 receptor agonism improves metabolic, biochemical, and histopathological indices of nonalcoholic steatohepatitis in mice. Am J Physiol Gastrointest Liver Physiol 2012;302:G762-72.

18. Liu Y, Wei R, Hong TP. Potential roles of glucagon-like peptide-1-based therapies in treating non-alcoholic fatty liver disease. World J Gastroenterol 2014;20:9090-7.

19. Jendle J, Nauck MA, Matthews DR, Frid A, Hermansen K, Düring $M$, et al. Weight loss with liraglutide, a once-daily human glucagon-like peptide-1 analogue for type 2 diabetes treatment as monotherapy or added to metformin, is primarily as a result of a reduction in fat tissue. Diabetes Obes Metab 2009;11:1163-72.

20. Ter Horst KW, Serlie MJ. Fructose consumption, lipogenesis, and non-alcoholic fatty liver disease. Nutrients 2017;9:981.

21. Bifari F, Manfrini R, Dei Cas M, Berra C, Siano M, Zuin M, et al. Multiple target tissue effects of GLP-1 analogues on nonalcoholic fatty liver disease (NAFLD) and non-alcoholic steatohepatitis (NASH). Pharmacol Res 2018;137:219-29.

22. Parlevliet ET, Wang Y, Geerling JJ, Schröder-Van der Elst JP, Picha K, O'Neil K, et al. GLP-1 receptor activation inhibits VLDL production and reverses hepatic steatosis by decreasing hepatic lipogenesis in high-fat-fed APOE*3-Leiden mice. PLoS One 2012;7:e49152.

23. Zhang L, Yang M, Ren H, Hu H, Boden G, Li L, et al. GLP-1 analogue prevents NAFLD in ApoE KO mice with diet and Acrp30 knockdown by inhibiting c-JNK. Liver Int
2013;33:794-804.

24. Zhou D, Lezmi S, Wang H, Davis J, Banz W, Chen H. Fat accumulation in the liver of obese rats is alleviated by soy protein isolate through $\beta$-catenin signaling. Obesity (Silver Spring) 2014;22:151-8.

25. Seo MH, Lee J, Hong SW, Rhee EJ, Park SE, Park CY, et al. Exendin-4 inhibits hepatic lipogenesis by increasing $\beta$-catenin signaling. PLoS One 2016;11:e0166913.

26. Ip W, Shao W, Chiang YT, Jin T. GLP-1-derived nonapeptide GLP-1(28-36)amide represses hepatic gluconeogenic gene expression and improves pyruvate tolerance in high-fat dietfed mice. Am J Physiol Endocrinol Metab 2013;305:E134858.

27. Abiola M, Favier M, Christodoulou-Vafeiadou E, Pichard AL, Martelly I, Guillet-Deniau I. Activation of Wnt/beta-catenin signaling increases insulin sensitivity through a reciprocal regulation of Wnt10b and SREBP-1c in skeletal muscle cells. PLoS One 2009; 4:e8509.

28. Xu H, Wang J, Zhang X, Li Z, Wang Y, Xue C. Inhibitory effect of fucosylated chondroitin sulfate from the sea cucumber Acaudina molpadioides on adipogenesis is dependent on Wnt/ $\beta$-catenin pathway. J Biosci Bioeng 2015;119:85-91.

29. Zhai X, Yan K, Fan J, Niu M, Zhou Q, Zhou Y, et al. The $\beta$ catenin pathway contributes to the effects of leptin on SREBP$1 \mathrm{c}$ expression in rat hepatic stellate cells and liver fibrosis. $\mathrm{Br}$ J Pharmaco. 2013;169:197-212.

Received for publication: 14 July 2020. Accepted for publication: 5 September 2020.

This work is licensed under a Creative Commons Attribution-NonCommercial 4.0 International License (CC BY-NC 4.0).

(C) Copyright: the Author(s), 2020

Licensee PAGEPress, Italy

European Journal of Histochemistry 2020; 64:3160

doi:10.4081/ejh.2020.3160 